\title{
La structure des verres étudiée par diffraction des neutrons
}

\author{
L. Cormier
}

\author{
Laboratoire de Minéralogie-Cristallographie, Universités Paris 6 et 7, UMR 7590 du CNRS, \\ Institut de Physique du Globe, 4 place Jussieu, 75005 Paris, France
}

\begin{abstract}
Résumé : La diffraction des neutrons est une méthode largement utilisée pour déterminer la structure des matériaux amorphes et en particulier des verres. L'utilisation de la méthode de substitution isotopique permet d'extraire les fonctions de distribution de paires partielles centrées autour d'un élément choisi. Nous présentons quelques exemples récents d'études par diffraction des neutrons sur des verres qui ont permis de mieux comprendre à la fois le réseau polymérique de la matrice vitreuse et l'environnement local et à moyenne distance autour des cations. Ces études ont révélées un ordre structural s'étendant au delà des premiers voisins, jusque vers de distances d'environ $10 \AA$. Le couplage avec d'autres méthodes expérimentales (diffraction anomale des rayons $X$ ) et des techniques de simulations (dynamique moléculaire, Monte Carlo Inverse ou RMC) sont indispensables pour affiner nos connaissances de la structure des verres.
\end{abstract}

\section{INTRODUCTION}

L'état vitreux est un état métastable, intermédiaire entre le cristal et le liquide. Sa structure est souvent considérée comme étant proche de celle du liquide à un instant donné: c'est une "photographie instantanée" de la structure du liquide. A la différence des liquides, il n'existe pas de déplacement atomique dans les verres. Les atomes peuvent néanmoins vibrer, plus ou moins harmoniquement, autour de leurs sites (désordonnés) d'équilibre, si bien que la dynamique du verre est caractéristique d'un solide.

L'étude de la structure des verres touchent un nombre important de domaines :

- En physique, les propriétés fondamentales des verres diffèrent des lois applicables pour les solides cristallins qui reposent souvent sur la périodicité cristalline (théorème de Bloch, notion de phonons, structure de bandes). Ces propriétés, encore insuffisamment comprises du point de vue fondamental, dépendent largement de la structure à courte et grande distance du verre.

- En sciences des matériaux, dans l'industrie verrière (vitrage: bâtiments, pare-brise d'automobiles; emballages et conditionnement; fibres de renforcement et d'isolation; matériaux d'optique...) ou dans les arts (vitraux, vases...), les verres présentent des propriétés intéressantes aussi bien par leur formage et leur formulation que par leurs propriétés d'usage (coloration, isolation, résistance mécanique, expansion thermique, propriétés de surface...). Le contrôle de ces propriétés nécessitent une bonne connaissance de la structure microscopique.

- en Sciences de la Terre, la possibilité d'une analogie verre-liquide permet l'étude structurale de systèmes silicatés à hautes températures, représentatifs des liquides magmatiques, qui sont difficilement à étudier in situ. Les verres géologiques (verre volcaniques ou d'impact météoritiques) ou les inclusions vitreuses sont également largement étudiés.

Dans ce cours, nous traiterons principalement des verres à liaisons covalentes (verres d'oxydes). 


\section{GENERALITES SUR LA STRUCTURE DES VERRES}

\subsection{Définition d'un verre}

Le manque de modèles structuraux précis vient du désordre atomique qui empêche une description absolue comme pour les composés cristallins. La structure d'un cristal parfait est entièrement décrite par la position des atomes à l'intérieur d'une cellule unitaire et par les symétries de translation permettant de reproduire cette cellule dans l'espace. Une description totale de la structure du verre nécessiterait la détermination des coordonnées de chaque atome dans le composé, une tâche bien entendu impossible et qui ne permettrait pas de connaître la structure d'un autre verre. Il est donc préférable de parler en terme statistique de la position des atomes et de déterminer les principes qui đéfinissent leur arrangement [1].

Un verre peut être défini comme un solide non-cristallin présentant une transition vitreuse $\left(\mathrm{T}_{g}\right)$, c'est à dire le passage du liquide surfondu au verre (Figure 1). Cette transition se caractérise par des variables thermodynamiques $(\mathrm{V}, \mathrm{S}, \mathrm{H})$ qui sont continues alors que les quantités dérivées $\left(C_{p}, \alpha\right)$ sont discontinues à $T_{g}$, sans qu'il soit cependant possible de parler de simple transition du second ordre.

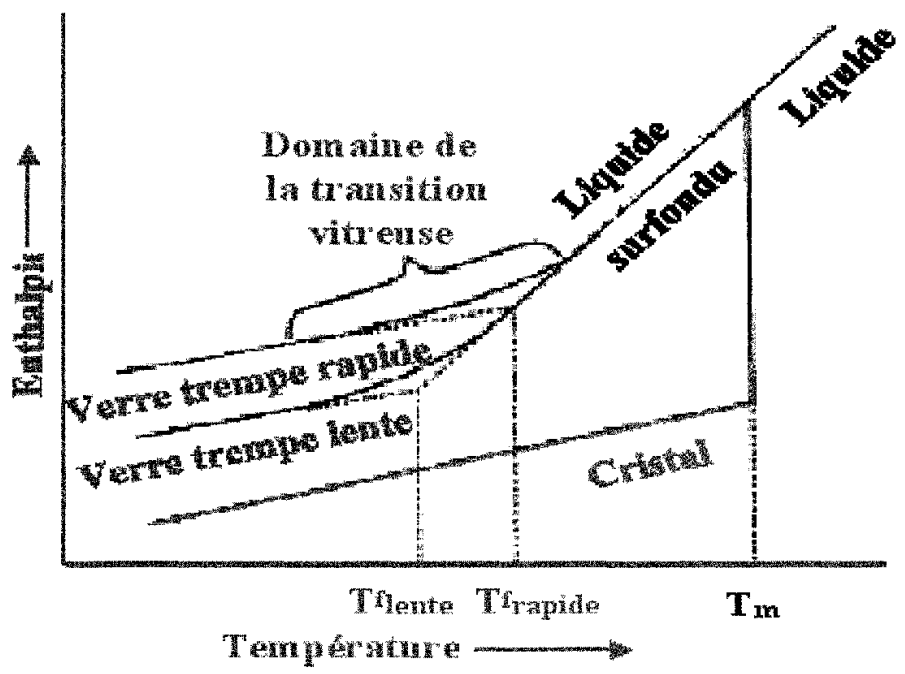

Figure 1: Variation de l'enthalpie en fonction de la température pour des vitesses de trempe différentes.

Le terme non-cristallin indique l'absence d'ordre à longue distance et de périodicité, qui résultent de variations dans les distances interatomiques et dans les angles de liaisons entre polyèdres (la position de deux atomes distants n'est pas corrélée). Le verre est obtenu (traditionnellement) par refroidissement rapide d'un liquide. On considère que c'est un solide macroscopique car il a une viscosité supérieure à $10^{13}$ Pa.s. Cette valeur est arbitraire mais c'est une définition largement utilisée qui correspond à des temps de relaxation de l'ordre de plusieurs minutes. De plus, cette valeur limite de $10^{13} \mathrm{~Pa}$.s permet également de définir la température de 
transition vitreuse, $\mathrm{T}_{\mathrm{g}}$, entre le liquide surfondu et le verre.

\subsection{Différentes échelles d'ordre}

Une meilleure compréhension de la structure des verres repose sur une meilleure connaissance de l'ordre à courte et à moyenne distance.

On distingue généralement trois types d'ordre dans les verres $[2,3]$ :

- l'ordre à courte distance correspondant aux polyèdres de coordinence et qui est caractérisé par la distance interatomiques, le nombre et la nature des premiers voisins, l'angle intra-polyédral, la géométrie et la symétrie du site;

- l'ordre à moyenne distance (5-20 ̊) impliquant:

- la nature des oxygènes premiers voisins, soit pontants $\left(\mathrm{O}_{\mathrm{p}}\right)$ soit non-pontants $\left(\mathrm{O}_{\mathrm{np}}\right)$ pour les verres d'oxydes;

- les types de connections (sommets, arêtes ou faces partagés) et les orientations relatives des polyèdres,

- les unités superstructurales (anneaux, clusters, comme par exemple l'anneau boroxol $\mathrm{B}_{3} \mathrm{O}_{6}$ dans les borates),

- la dimensionalité du réseau (2D ou 3D, par exemple la présence d'anneau boroxol favorise la formation d'arrangements planaires);

- l'ordre à longue distance qui concerne des fluctuations de densité ou de composition (séparation de phase)

\subsection{Modèles structuraux}

Les verres qui ont des liaisons dirigées et un caractère covalent important (silicates, borates, phosphates, germanates) sont décris par le modèle de Zachariasen [4]. Dans ce modèle aléatoire continu (Continuous Random Network, CRN) on distingue les éléments selon leur rôle dans la structure:

- les formateurs de réseau: ce sont des éléments fortement chargés qui forment des liaisons covalentes rigides avec les oxygènes pour donner des polyèdres de faible coordinence. Ces unités structurales élémentaires $\left(\mathrm{SiO}_{4}, \mathrm{BO}_{3}\right.$ par exemple) sont comparables à celles qui existent dans les cristaux. Elles forment un réseau tridimensionnel continu en se connectant par sommets. Les oxydes formateurs les plus courants sont $\mathrm{SiO}_{2}, \mathrm{~B}_{2} \mathrm{O}_{3}, \mathrm{GeO}_{2}, \mathrm{P}_{2} \mathrm{O}_{5}$;

- les non-formateurs de réseau: ce sont des éléments de plus grandes tailles, avec une charge faible, formant des polyèdres de grande coordinence. Ils s'incorporent dans le réseau soit en compensateurs de charge (en s'insérant alors dans les interstices), soit en modificateurs qui brisent les liaisons entre les polyèdres élémentaires et transforment des $O$ pontants $\left(O_{p}\right)$ en $O$ non-pontants $\left(\boldsymbol{O}_{\mathrm{np}}\right)$, dépolymérisant ainsi le réseau. Ce sont essentiellement les alcalins, les alcalino-terreux ou les éléments de transition;

- les intermédiaires, qui selon la composition du verre auront plutôt un rôle de formateur ou de modificateur: $\mathrm{Al}, \mathrm{Ti}, \mathrm{Fe}, \mathrm{Pb}$. En remplaçant des atomes de silicium, ils entrânent un déficit de charge qui sera compensé par des cations non-formateurs.

Dans ce modèle, les éléments modificateurs sont supposés s'insérer aléatoirement dans le réseau et avoir une première sphère de coordinence mal définie. Des études par EXAFS $[5,6]$ ont montré qu'en fait ces cations avaient une coordinence bien établie et souvent proche de celle existant dans les structures cristallines de composition similaire. La diffusion des neutrons sur des verres isotopiquement substitués a confirmé ces observations [7]. Elle a de plus permis, 
grâce aux travaux de Gaskell [8], de remettre en cause une distribution aléatoire de ces éléments dans le réseau polymérisé.

a)

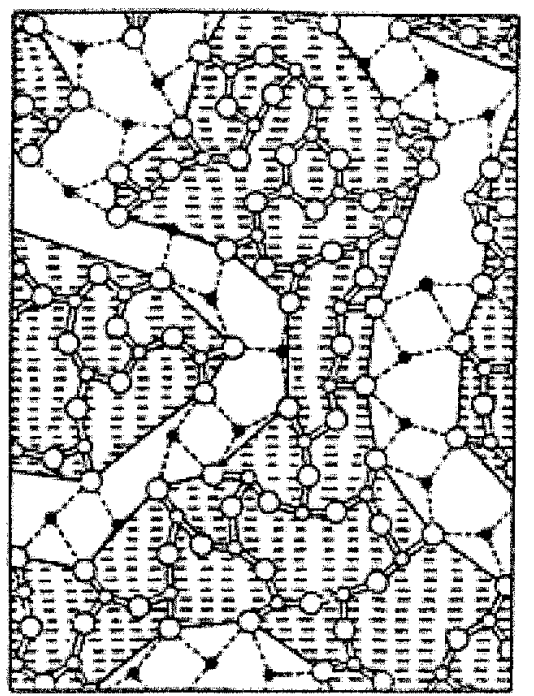

b)

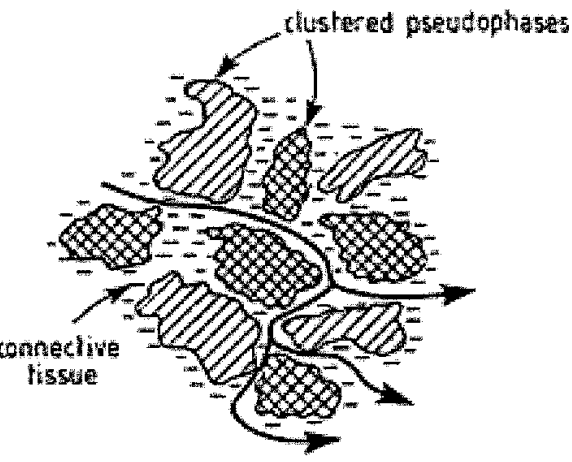

Figure 2: a) Représentation schématique du réseau aléatoire modifié de Greaves [9]. Les zones hachurées correspondent aux régions riches en formateurs et sont sillonnées par des canaux percolants contenant des cations modificateurs (atomes pleins noirs). b) Modèle proposé par Ingram montrant la présence de clusters et l'existence de chemins favorisant la migration des ions (flèches) à l'intérieur du tissu connectif [11].

Ces données nouvelles ont amené Greaves ${ }^{9}$ à proposer un nouveau modèle structural, le réseau aléatoire modifié (Random Modified Network. RMN), dans lequel les cations modificateurs ne sont pas distribués de façon homogène dans le réseau polymérisé (Figure 2a). Le verre est constitué de régions riches en formateurs qui sont séparées par des régions plus riches en modificateurs. Le succès de ce modèle s'explique par la possibilité qu'il offre de donner une explication simple aux phénomènes de diffusion ou de conduction ionique. A partir d'une concentration suffisante de modificateurs, les zones riches en modificateurs (qui peuvent être représentées comme des canaux sillonnant les régions riches en formateurs) se rejoignent au sein de la structure. Le réseau isolant silicaté est alors contourné et la conduction ionique peut être assurée par des ions de petites tailles $\left(\mathrm{Li}^{+}\right)$. La présence de cations plus volumineux dans ces canaux peut faire obstacle à la diffusion des ions de petites tailles et expliquer les changements de propriétés ioniques pour des mélanges d'alcalins.

Un autre modèle a également été proposé par Goodman [10] et Ingram [11] dans lequel des microdomaines très ordonnés (clusters ou pseudophases) sont ceinturés par un tissu de connexion très désordonné qui sert de passage pour la migration des ions. Dans ce modèle (Figure 2b) le tissu connectif est continu, il n'y a donc pas de seuil percolant pour la diffusion. Seules les contraintes topologiques restreignent les déplacements ioniques. 
Les verres de fluorures à base de $\mathrm{Zr}$, Hf ou Ba ne suivent pas les règles de Zachariasen car les cations ont des coordinences avec les atomes de fluor élevées (6 ou 8). Par contre les verres à base de $\mathrm{BeF}_{2}$ se comporte comme un réseau de tétraèdres $\mathrm{BeF}_{4}$ similaire à la silice avec des éléments additionnels jouant le rôle de modificateurs. De la même façon le verre $\mathrm{ZnCl}_{2}$ présente une structure à base de tétraèdres.

Les verres de chalcogénures (verres à base de $\mathrm{S}$, Se ou Te) se caractérisent par une structure plus complexes que les verres d'oxydes car des liaisons directes entre atomes de même type est possible. Leur structure est généralement composée d'anneaux ou de chaînes d'atomes. Des éléments adđitionnelles peuvent se positionner interstitiellement entre les chaînes et s'associer à un chalcogénure terminal, jouant alors un rôle de modificateur, comme dans les verres d'oxydes. Alternativement, As et $P$ peuvent se substituer dans les chaînes d'atomes et en augmenter la longueur (Figure 3). A faible concentration en As, Ge ou Si, ces atomes sont localisés aux points où les chaînes se branchent ou se croisent. Un modèle moléculaire pour les verres de chalcogénures d'arsenic correspond à un empilement aléatoire de molécules approximativement sphériques $\mathrm{As}_{4} \mathrm{X}_{4}$ et/ou $\mathrm{As}_{4} \mathrm{X}_{6}(\mathrm{X}=\mathrm{S}, \mathrm{Se}, \mathrm{Te})$. Il a été également proposé que ces verres présentent des clusters moléculaires avec une structure en couche, par exemple pour les verres $\mathrm{As}_{2} \mathrm{~T}_{3}$ ou $\mathrm{GeT}_{2}(\mathrm{~T}=\mathrm{Se}, \mathrm{S})$ [12].

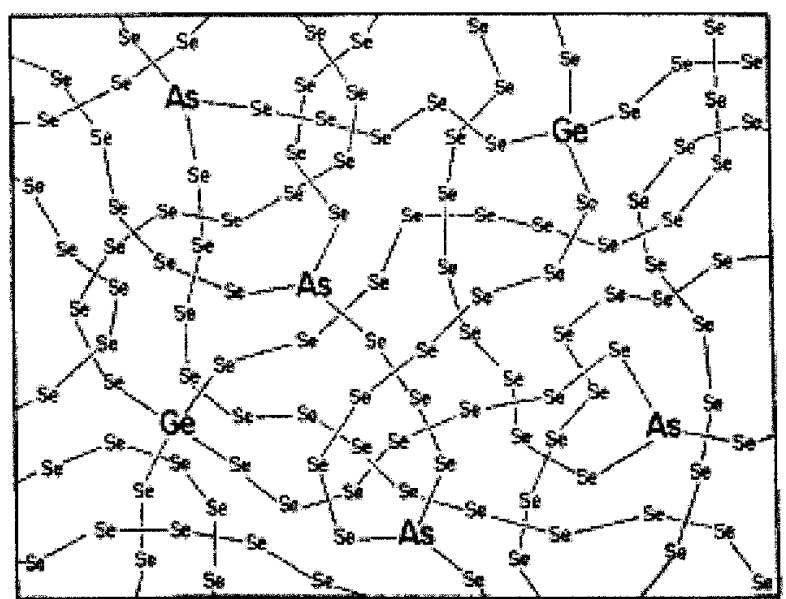

Figure 3: Modèle structural d'un verre de chalcogénures à base de chaînes de liaisons homopolaires Se-Se. Les éléments modificateurs (As, Ge) relie les chaînes de chalcogénures.

Pour des verres à liaisons non-dirigées (verres métalliques ou ioniques), le modèle le mieux adapté repose sur un empilement compact aléatoire de sphères (Figure 4). Bien que les liaisons dans ces verres ne soient pas dirigées par les orbitales électroniques, un ordre local chimique et topologique est possible (empilement trigonal prismatique proposé pour les verres métaux de transition-métalloides ou icosaèdre). Cet arrangement à courte distance pourrait être une conséquence de corrélations structurales à moyenne distance [13]. La nature de la première couche de coordinence, le rapport des rayons atomiques, la densité d'empilement sont des paramètres permettant de définir la structure de ces verres. 


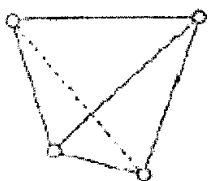

ii:

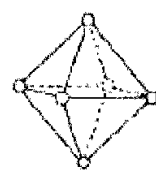

(1)

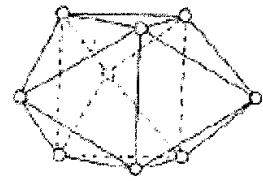

(ii)

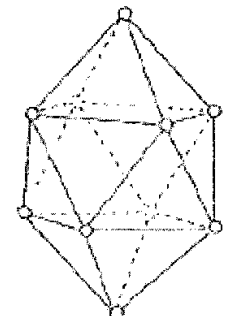

sw

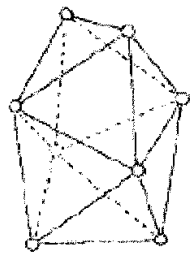

iv)

Figure 4: Cavités canoniques de Bernal déterminées pour un empilement compact aléatoire de sphères dures de taille unique. (i) tétrahèdre ; (ii) octahèdre ; (iii) prisme trigonal ; (iv) anti-prisme d'Archimède ; (v) dodécahèdre tetragonal

\section{RAPPELS SUR LA THEORIE DE LA DIFFRACTION}

La diffraction des neutrons par des solides amorphes ou des liquides présentent des différences importantes avec la diffraction des rayons X [14]. Alors que les rayons X sont diffusés par les électrons, les neutrons non-chargés interagissent directement avec le noyau. En conséquence, ils peuvent être utilisés pour étudier la position structural d'éléments légers comme $\mathrm{H}$ ou Li. L'interaction avec un atome donné ne varie pas de manière simple avec $\mathrm{Z}$, comme c'est le cas pour les rayons $\mathrm{X}$, mais peut être très différente entre deux éléments voisins et même entre isotopes d'un même élément. Cette dernière propriété est à la base de la méthode de substitution isotopique expliquée ci dessous.

\subsection{Le facteur de structure et la fonction de corrélation}

Lors d'une expérience conventionnelle de diffraction totale, les neutrons sont diffractés élastiquement et inélastiquement (résultant de l'interaction du faisceau de neutrons avec les vibrations thermiques). Par une mesure de diffraction totale, on obtient une information sur la structure statique du verre qui est contenue par le facteur de structure, $S(Q)$, avec $Q$ l'amplitude du vecteur de diffraction, défini par $Q=4 \pi \sin \theta / \lambda$ ( $2 \theta$ est l'angle de diffraction et $\lambda$ la longueur d'onde des neutrons). La transformée de Fourier du facteur de structure donne une fonction de corrélation, $\mathrm{G}(\mathrm{r})$, qui décrit les distances inter atomiques dans l'espace réel.

Pour des matériaux isotropes comme les verres et monoatomiques, le facteur de structure peut s'écrire [15]:

$$
\mathrm{S}(\mathrm{Q})=1+4 \pi \rho_{0} \int_{0}^{\infty} \mathrm{r}^{2}[\mathrm{~g}(\mathrm{r})-1] \frac{\sin (\mathrm{Q} r)}{\mathrm{Qr}} \mathrm{dr}
$$

où $r$ est la distance inter atomique, $\rho_{0}$ (en atomes $\AA^{-3}$ ) est la densité atomique moyenne et

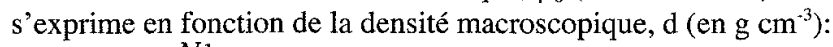

$$
\rho_{0}=\frac{N d}{A^{*} 10^{24}}
$$

$\mathrm{N}$ étant le nombre d'Avogadro et $\mathrm{A}$ la masse atomique de l'échantillon. 
La fonction de corrélation de paire, g(r), décrit les fluctuations locales de densité autour de l'unité.

Pour des matériaux polyatomiques, le facteur de structure total (la fonction de corrélation) est une somme pondérée de tous les facteurs de structure partiels, $S_{\alpha \beta}(Q)$ ), (toutes les fonctions de distribution de paires partielles, $\left.\mathrm{g}_{\alpha \beta}(\mathrm{r})\right)[16]$ :

$$
\begin{aligned}
& S(Q)=\sum_{\alpha \beta} W_{\alpha \beta} S_{\alpha \beta}(Q) \\
& G(r)=4 \pi r \rho_{0} \sum_{\alpha \beta} W_{\alpha \beta}\left[g_{\alpha \beta}(r)-1\right]
\end{aligned}
$$

où $W_{\alpha \beta}$ sont les facteurs pondérant, $W_{\alpha \beta}=c_{\alpha} c_{\beta} b_{\alpha} b_{\beta}$, et $c_{\alpha}$ et $b_{\alpha}$ sont respectivement les concentrations atomiques et la longueur de diffusion des neutrons pour l'atome de type $\alpha . \mathrm{g}_{\alpha \beta}(\mathrm{r})$ donne la probabilité de trouver un atome de type $\beta$ à la distance r quand un atome de type $\alpha$ est à l'origine (distribution moyenne des atomes $\beta$ autour d'un atome $\alpha$ à l'origine).

Plusieurs fonctions peuvent être utilisées pour décrire la structure : la fonction de corrélation de paire réduite, $T(r)=4 \pi r \rho_{0} \sum_{\alpha \beta} W_{\alpha \beta} g_{\alpha \beta}(r)$, et la fonction de distribution radiale, $\operatorname{RDF}(r)=4 \pi r^{2} \rho_{0} \sum_{\alpha \beta} W_{\alpha \beta} g_{\alpha \beta}(r)$.

Ces fonctions sont similaires et donnent une représentation uni-dimensionelle de la structure tri-dimensionelle du matériau étudié.

Les données expérimentales sont limitées en $\mathrm{Q}(\mathrm{Q} \leq \mathrm{Q}$ max $)$. La transformée de Fourier du facteur de structure n'est donc pas intégrable jusqu'à l'infini ce qui entraîne l'apparition de lobes parasites autour des pics, particulièrement à bas $\mathrm{r}$. Cette troncation revient à multiplier la fonction d'interférence $\mathrm{Q}(\mathrm{S}(\mathrm{Q})-1)$ par une fonction de modification $\mathrm{M}(\mathrm{Q})$, qui décroît lentement jusqu'à 0 à $\mathrm{Q}_{\max }$ :

$$
\mathrm{G}(\mathrm{r})=\frac{2}{\pi} \int_{0}^{\infty} \mathrm{Q}(\mathrm{S}(\mathrm{Q})-1) \mathrm{M}(\mathrm{Q}) \sin (\mathrm{rQ}) \mathrm{dQ}
$$

La fonction de modification généralement utilisée est la fonction de Lorch [17] :

$$
M\left(Q_{0}\right) \begin{cases}=\frac{\sin \left(\Delta r Q_{0}\right)}{\Delta r Q_{0}} & Q_{0} \leq Q_{\max } \\ =0 & Q_{0}>Q_{\max }\end{cases}
$$

avec $\Delta r=\pi / Q_{\max }$, qui représente la longueur de la résolution dans l'espace réel.

L'introduction de cette fonction équivaut à faire une convolution de la fonction de corrélation :

$$
G^{\prime}(r)=\int_{0}^{\infty} G(r) P(r-u) d u
$$

avec la fonction $\mathrm{P}(\mathrm{r})$ définie par :

$$
\mathrm{P}(\mathrm{r})=\int_{0}^{\mathrm{Q}_{\max }} \frac{\sin (\Delta \mathrm{rQ})}{\Delta \mathrm{rQ}} \cos (\mathrm{rQ}) \mathrm{dQ}
$$

La convolution va permettre d'enlever les oscillations parasites, mais au détriment de la finesse des pics dans $\mathrm{G}(\mathrm{r})$. La fonction $\mathrm{P}(\mathrm{r})$ présente un pic à $\mathrm{r}=0$ qui est entouré de "vaguelettes". La largeur à mi-hauteur du pic vaut $2 \Delta \mathrm{r}=5.437 / \mathrm{Q}_{\max }$ et son intensité $0.1876 \mathrm{Q}_{\max }$. L'effet de cette fonction est donc réduit si on peut avoir accès expérimentalement à de grandes valeurs de $\mathrm{Q}_{\max }$.

L'ajustement des pics expérimentaux se fait généralement avec des fonctions gaussiennes : 


$$
\mathrm{g}_{\alpha \beta}(\mathrm{r})=\frac{\mathrm{W}_{\alpha \beta \mathrm{n}_{\beta}}}{\mathrm{c}_{\alpha} \mathrm{r} \sqrt{2 \pi \Delta_{\alpha \beta}^{2}}} \cdot \mathrm{e}^{-\left(\mathrm{r}-\mathrm{r}_{\alpha \beta}\right)^{2} / 2 \Delta_{\alpha \beta}^{2}}
$$

Trois paramètres peuvent varier : la distance entre l'atome à la distance $r_{\alpha}$ et l'atome $\alpha$ pris comme origine, le facteur de Debye-Waller $\left(\Delta_{\alpha \beta}\right)$ et le nombre de voisins de type $\beta\left(n_{\beta}\right)$ à la distance $r_{\alpha}$. La distance interatomique $r_{\alpha}$ est déterminée avec une excellente précision. Par contre, la coordinence et le facteur de Debye-Waller sont étroitement corrélés et sujet à plus d'incertitudes. Le nombre de coordinence $n_{\beta}$ est le paramètre déterminé avec la plus faible précision, car il varie fortement avec la pente à l'origine ou le domaine en $\mathrm{Q}$, et la précision diminue bien sûr si les pics se recouvrent. La possibilité d'utiliser différentes fonctions (totales ou partielles), qui doivent donner une même information, peut apporter des indications sur la précision des valeurs trouvées. Pour tenir compte de la troncation à $\mathrm{Q}_{\max }$, les gaussiennes utilisées sont convoluées par une fonction de résolution dans l'espace réel donnée par la transformée de Fourier de la fonction de la fonction Lorch.

Pour les neutrons, les longueurs de diffusion sont indépendantes de $\mathrm{Q}$ alors que le facteur de diffusion en rayons $\mathrm{X}$ décrô̂t vers zéro à grandes valeurs de $\mathrm{Q}$. Non seulement cette propriété aide à la réduction des données, mais un large domaine en $\mathrm{Q}$ est accessible par diffraction des neutrons $\left(0-50 \AA^{-1}\right)$, ce qui permet une détermination directe à la fois de l'ordre local et à moyenne distance avec une grande précision. Bien que l'absorption des rayons $\mathrm{X}$ peut être utilisé pour des niveaux de concentration très faible ou pour détecter des teneurs mineures de sites, les nombres de coordinence et les distances inter atomiques sont moins bien évaluées que par diffraction des neutrons car les faibles valeurs en $\AA^{-1}$ ne peuvent pas être utilisé [6]. Les rayons X de grande énergie permettent d'accéder à un domaine en $\mathrm{Q}\left(0.8-32 \AA^{-1}\right)$ comparable aux neutrons mais le rapport signal sur bruit est plus faible et les corrections sont moins bien comprise par la théorie que dans le cas de la diffraction des neutrons [18]. De plus une normalisation absolue en utilisant une référence de vanadium est possible en diffraction des neutrons. Cependant pour des matériaux présentant une forte inélasticité ou des corrections d'absorption importantes (par exemple, $\mathrm{Li}$ ou $\mathrm{H}$ ), des précautions sont nécessaires dans l'analyse des données de diffraction de neutrons.

\subsection{La méthode de substitution isotopique}

La fonction $\mathrm{G}(\mathrm{r})$ est riche en informations mais elle est dominée par les corrélations qui ont une grande pondération (grand $\mathrm{W}_{\alpha \beta}$ ), c'est-à-dire généralement $\mathrm{Si}-\mathrm{O}, \mathrm{O}-\mathrm{O}, \mathrm{Si}-\mathrm{Si}$ dans les silicates. D'autre part, au delà de $3 \AA$, les différentes contributions se superposent, les contributions avec de petits facteurs de pondération étant recouvertes par celles avec de grands facteurs de pondération. Il devient extrêmement difficile au-delà de $3 \AA$ de décorréler les différentes contributions dans un matériau multicomposant. Le recours à des modèles structuraux peut permettre de proposer une interprétation de $G(r)$ mais elle n'est bien sûr pas univoque.

De fait, idéalement, on souhaiterait extraire chaque fonction de corrélation partielle $\mathrm{g}_{\alpha \beta}(\mathrm{r})$. Pour un matériau composé de $n$ éléments, il faudrait réaliser $n(n+1) / 2$ expériences différentes, ce qui devient rapidement impossible avec des verres multicomposants. Le seul élément dans $\mathrm{S}(\mathrm{Q})$ qui ne dépende pas de la structure est la longueur de diffusion, $b$. Celle-ci peut varier de façon importante d'un élément à l'autre ou entre les isotopes d'un même élément. La première propriété est utilisée dans des substitutions isomorphiques (échange d'éléments jouant un rôle identique 
dans la structure) alors que la seconde conduit à une méthode plus rigoureuse de contraste, la substitution isotopique.

La méthode de substitution isotopique a été utilisée pour la première fois par Enderby [19], pour extraire les trois facteurs de structure partiels d'un alliage liquide $\mathrm{Cu}-\mathrm{Sn}$. De nombreuses études de substitution isotopique ont été menées ces dernières années $[8,20]$, bénéficiant notamment du développement de nouvelles sources de neutrons. Cette technique repose sur la mesure de la diffraction des neutrons par deux composés, préparés de façon rigoureusement identique (on suppose donc qu'ils ont la même structure) mais avec des compositions isotopiques différentes pour l'élément $M$. On obtient deux facteurs de structure totaux dont seuls les poids des facteurs de structure partiels incluant $M$ vont varier :

$$
\begin{aligned}
& S(Q)=\sum_{\substack{\alpha \beta \\
\alpha, \beta \neq M}} c_{\alpha} c_{\beta} b_{\alpha} b_{\beta}\left(S_{\alpha \beta}(Q)-1\right)+\sum_{\substack{\alpha M \\
\alpha \neq M}} c_{\alpha x} c_{M} b_{\alpha} b_{M}\left(S_{\alpha M}(Q)-1\right)+c_{M}^{2} b_{M}^{2}\left(S_{M M}(Q)-1\right) \\
& S^{\prime}(Q)=\sum_{\substack{\alpha, \beta \neq M \\
n_{\alpha}}} c_{\alpha} c_{\beta} b_{\alpha} b_{\beta}\left(S_{\alpha \beta}(Q)-1\right)+\sum_{\substack{\alpha M \\
\alpha \neq M}} c_{\alpha} c_{M} b_{\alpha} b_{M}^{\prime}\left(S_{\alpha M}(Q)-1\right)+c_{M}^{2} b_{M}^{\prime 2}\left(S_{M M}(Q)-1\right)
\end{aligned}
$$

La technique de différence consiste à soustraire ces deux quantités et donc à éliminer tous les termes ne comprenant pas $M$. On obtient la fonction dite de première différence, $\Delta_{M}(Q)$, qui est la somme des facteurs de structure partiels centrés sur l'élément $M$. En faisant cette soustraction, on obtient directement la fonction de corrélations de paires centrées sur $\mathrm{M}$ :

$$
\begin{aligned}
& \mathrm{G}_{M-\alpha}(\mathrm{r})=\frac{2}{\pi} \int \Delta_{M}(\mathrm{Q}) \mathrm{Q} \sin (\mathrm{Q}) \mathrm{dQ} \\
& \quad=2 \sum_{\alpha \neq M} \mathrm{c}_{\alpha} \mathrm{c}_{M} \mathrm{~b}_{\alpha}\left(\mathrm{b}_{\mathrm{M}}-\mathrm{b}_{\mathrm{M}}^{\prime}\right) \mathrm{G}_{\alpha M}(\mathrm{r})+\mathrm{c}_{\mathrm{M}}^{2}\left(\mathrm{~b}_{\mathrm{M}}^{2}-\mathrm{b}_{\mathrm{M}}^{\prime 2}\right) \mathrm{G}_{M M}(\mathrm{r})
\end{aligned}
$$

On a ainsi une sonde chimiquement sélective. La fonction obtenue est analogue à la différence calculée par diffraction anomale des rayons $\mathrm{X}$ (voir section 3.3) ou à la transformée de Fourier du signal EXAFS.

Il est également possible de mesurer trois échantillons et d'obtenir ainsi deux premières différences, $\Delta_{\mathrm{M}}(\mathrm{Q})$ et $\Delta_{\mathrm{M}}^{\prime}(\mathrm{Q})$ :

$$
\begin{aligned}
& \Delta_{M}(Q)=2 \sum_{\alpha \neq M} c_{\alpha} c_{M} b_{\alpha}\left(b_{M}-b_{M}^{\prime}\right)\left(s_{\alpha M}(Q)-1\right)+c_{M}^{2}\left(b_{M}^{2}-b_{M}^{\prime 2}\right)\left(S_{M M}(Q)-1\right) \\
& \Delta_{M}^{\prime}(Q)=2 \sum_{\alpha \neq M} c_{\alpha} c_{M} b_{\alpha}\left(b_{M}^{\prime}-b_{M}^{\prime \prime}\right)\left(s_{\alpha M}(Q)-1\right)+c_{M}^{2}\left(b_{M}^{\prime 2}-b_{M}^{\prime \prime 2}\right)\left(S_{M M}(Q)-1\right) .
\end{aligned}
$$

On constate que les facteurs pondérants dépendent de la différence des longueurs de diffusion de $M$ pour les termes $M-\alpha(\alpha \neq M)$ alors que le terme M-M dépend de la différence du carré des longueurs de diffusion. Il est donc possible, par un choix judicieux de la composition isotopique des trois verres, d'égaliser les quantités $\Delta b=b_{M}-b_{M}^{\prime}$ et $\Delta b^{\prime}=b_{M}^{\prime}-b_{M}^{\prime \prime}$. Cette condition est aisément remplie si:

$$
\mathrm{b}^{\prime}=\frac{\mathrm{b}+\mathrm{b}^{\prime \prime}}{2} \text {. }
$$


La soustraction de ces deux fonctions va donner la fonction de seconde différence, $\Delta\left(\Delta_{\mathrm{M}}(\mathrm{Q})\right)$, dans laquelle n'est conservée que la corrélation M-M:

$$
\Delta\left(\Delta_{M}(\mathrm{Q})\right)=\frac{1}{2} c_{M}^{2}\left(b_{M}-b_{M}^{\prime \prime}\right)^{2}\left(S_{M M}(Q)-1\right)
$$

Cette fonction est bien sûr reliée à une fonction de corrélation de paire qui décrit directement la distribution de l'élément $M$ dans la structure:

$$
\begin{gathered}
\mathrm{G}_{\mathrm{MM}}(\mathrm{r})=\frac{2}{\pi} \int \Delta\left(\Delta_{\mathrm{M}}(\mathrm{Q})\right) \mathrm{Q} \sin (\mathrm{Qr}) \mathrm{dQ} \\
=\mathrm{c}_{\mathrm{M}}^{2} \frac{\left(\mathrm{b}_{\mathrm{M}}-\mathrm{b}_{\mathrm{M}}^{\prime \prime}\right)^{2}}{2} \mathrm{~g}_{\mathrm{MM}}(\mathrm{r})
\end{gathered}
$$

Bien que la diffraction anomale des rayons $\mathrm{X}$ (voir prochaine section) puisse être obtenue à trois énergies différentes, le contraste permettant d'extraire une fonction partielle M-M est faible et cette méthode a été peu utilisée. Un exemple récent est l 'étude de la distribution du Ba dans des verres silicatés [21].

Les limites de la technique de diffraction des neutrons avec substitution isotopique sont tout d'abord le nombre restreint d'éléments présentant des isotopes qui conviennent; les rayons $\mathrm{X}$ permettent d'accéder à plus d'éléments mais ils ne peuvent pas sonder les éléments légers comme l'hydrogène ou le lithium. Il faut que la différence entre les longueurs de diffusion des isotopes soit suffisamment grande, disons $\Delta \mathrm{b}>3 \mathrm{fm}$, pour obtenir une première et une seconde différence. Un $\Delta b$ plus faible peut donner accès seulement à une première différence, surtout pour des concentrations atomiques supérieures à $3 \%{ }^{1}$. Ces isotopes doivent être stables, ne pas être trop absorbants. Une contrainte supplémentaire est la disponibilité et le coût des différents isotopes, ce qui limite le nombre d'éléments accessibles à cette approche (des isotopes avec de faibles concentrations naturelles, $\mathrm{c}<1 \%$, ont un prix trop prohibitif pour être utilisés), d'autant que les expériences sont menées avec typiquement $4-7 \mathrm{~g}$ de chaque spécimen.

De nombreuses erreurs peuvent limiter sérieusement la précision (et même la justesse) des fonctions de différences. Cette méthode, au contraire de la diffraction anomale des rayons X, utilise plusieurs spécimens synthétisés dans les mêmes conditions et dont on suppose qu'ils ont la même structure. Chaque échantillon doit être parfaitement identique à l'exception de la composition isotopique de $\mathrm{M}$. Cela signifie que les concentrations de chaque élément doivent être parfaitement connues. Les rapports isotopiques de $M$ doivent aussi être déterminés de façon à pouvoir évaluer correctement $b_{M}$ pour chaque échantillon (bien que les longueurs de diffusion ne soient pas toujours connues avec précision). Les erreurs peuvent donc rapidement s'accumuler et contaminer sérieusement la seconde différence, rendant son interprétation délicate.

La précision statistique des données doit correspondre approximativement à $10^{6}$ coups par point [21] pour avoir une précision d'environ $1 \%{ }^{2}$. La première différence a l'avantage de réduire les problèmes liés aux erreurs systématiques: les corrections d'inélasticité, de diffusion

\footnotetext{
${ }^{1}$ L'étude d'éléments faiblement dilués, et bien sûr de composés naturels, ne peut donc pas être ré alisée par cette méthode, au contraire de l'EXAFS qui présente à cet égard un avantage certain. ${ }^{2}$ L'incertitude du détecteur pour $n$ événements comptés est $\sqrt{n}$. Une précision de $1 \%$ signifie qu e la dispersion statistique du signal est inférieure à $1 \%$.
} 
multiple sont en grande partie éliminées lors de la soustraction. Cependant il faut avoir conscience que ces termes réapparaissent dans la seconde différence.

\subsection{Diffraction anomale des rayons $X$}

La diffraction des rayons $X$ est largement utilisée comme méthode complémentaire à la diffraction des neutrons (voir section 5.1) et elle peut également permettre de déterminer des fonction des de distribution partielles avec une méthode de différence.

Le facteur de structure total déterminé par diffraction des rayons $\mathrm{X}$ est a somme de tous les facteur de structure partiels :

$$
\mathrm{S}(\mathrm{Q})=\sum_{\alpha, \beta} \mathrm{W}_{\alpha \beta}(\mathrm{Q}) \mathrm{S}_{\alpha \beta}(\mathrm{Q})
$$

mais, contrairement aux neutrons, les facteurs pondérant sont dépendant en $\mathrm{Q}$ :

$$
\mathrm{W}_{\alpha \beta}(\mathrm{Q})=\frac{\mathrm{c}_{\alpha} \mathrm{c}_{\beta} \Re\left(\mathrm{f}_{\alpha}(\mathrm{Q}, \mathrm{E})_{\beta}^{*}(\mathrm{Q}, \mathrm{E})\right)}{\mid \mathrm{f}(\mathrm{Q}, \mathrm{E})) \mid}\left(2-\delta_{\alpha \beta}\right)
$$

où $\mathrm{f}_{\alpha}$ est le facteur de diffusion atomique pour l'atome de type a qui dépend du vecteur de diffusion, $\mathrm{Q}$, et de l'énergie des rayons $\mathrm{X}, \mathrm{E}$.

Cette dépendance conduit à une expression de la fonction de corrélation plus compliquée que dans le cas des neutrons :

$$
\mathrm{G}(\mathrm{r})=\sum_{\substack{\alpha \beta \\ \beta>\alpha}} \mathrm{TF}\left(\mathrm{w}_{\alpha \beta}\right) * \mathrm{G}_{\alpha \beta}(\mathrm{r})
$$

avec TF la transformée de Fourier des facteurs pondérant et le symbole * signifie une convolution entre les deux termes.

Il est donc possible de faire varier le facteur de diffusion pour un élément en mesurant le même verre à deux énergies différentes, l'une proche d'un seuil d'absorption (où la variation de f est importante) et une autre plusieurs centaines d'eV au-dessous ou au-dessus du seuil. Les mesures se font au-dessous du seuil pour éviter les problèmes d'absorption et de fluorescence. La soustraction des deux intensités mesurées permet d'obtenir une fonction de première différence :

$$
\Delta_{M}(Q)=\sum_{\beta} D W_{M \beta}(Q) S_{M \beta}(Q)
$$

avec $D W_{M \beta}$ le facteur pondérant différentiel pour la paire $M-\beta$ :

$$
D W_{M \beta}=\frac{c_{M} c_{\beta} D\left(f_{M} f_{\beta}^{*}+f_{M}^{*} f_{\beta}\right)}{D\left(|(f)|^{2}\right)}
$$

La fonction différentielle permet de limiter l'information à l'environnement autour de l'élément considéré, M. Mais cette limitation n'est pas un handicap puisqu'elle elle permet d'extraire un signal qui est recouvert dans les fonctions de corrélations totales par des corrélations plus fortes, principalement en provenance du réseau vitreux. Une diffraction 
anomale peut également être obtenue pour certaines énergies par diffraction des neutrons [22]. Néanmoins cela ne concerne que quelques éléments qui présentent surtout un intérêt fondamental.

\section{EXEMPLES D'ETUDES DE VERRES PAR DIFFRACTION DES NEUTRONS}

\subsection{Les structures à bas $Q$}

Dans les facteurs de structure (de neutron et rayons X), il est souvent utile d'examiner la partie à faible valeur de $Q$ avant de faire une transformée de Fourier. Cette région est souvent dominée par un pic appelé « First Sharp Diffraction Peak» (FSDP) mais plusieurs pics ou épaulements peuvent coexister dans les verres chimiquement complexes. Les structures observées sont reliées à l'ordre à moyenne distance dans les verres mais ne donnent pas une structure bien localisée dans l'espace réel. Les dépendances en pression [24,25], température [26,27] et composition [28] de cette partie du facteur de structure sont souvent très différente du comportement du reste du facteur de structure. Bien qu'essentiel à la compréhension de l'ordre à moyenne distance, l'origine du ou des pics présents dans cette région est sujet à controverse [29-32].

Les explications proposés pour l'origine de ces pics considèrent principalement soit une organisation quasi-cristalline ou des corrélations entre des clusters et des vides. Price et Moss [29] ont suggéré une explication basée sur l'empilement des unités de base structurale ou moléculaire et une organisation atomique à moyenne distance, ce qui est effectivement observé dans certains composés moléculaire tétraédriques [33]. Un problème est que ces clusters sont mal définis et que la structure entre ces clusters n'est pas spécifiée. Un autre modèle propose la présence de zones de faibles occupations atomiques, autour de $5 \AA$ [34]. Blétry [35] a proposé un modèle de trous pour les structures vitreuses tétravalentes qui a été utilisé par Elliott pour une interprétation plus générale [30]. Elliott considère que ces structures sont issues de l'organisation chimique à courte distance des interstices autour des clusters centrés sur les cations (qui peuvent être par exemple les tétraèdres $\mathrm{SiO}_{4}$ dans les silicates). Le comportement en pression, température et composition peut être expliqué qualitativement par ce modèle.

La position de ces structures à bas Q coïncide souvent avec de forts pics de Bragg dans les cristaux de composition voisines. Il a été proposé que ces pics proviendrait d'un élargissement des réflexions de Bragg correspondant à des espacements périodiques anisotropes de longueur de cohérence limité dans l'espace réel $[31,36]$, comme des structures en couches qui existe dans les verres chalcogénures [25]. Les verres peuvent aussi présentés ces structures avec des intensités parfois plus importantes que dans les verres. Comme il semble improbable que le liquide soit plus organisé que le verre, l'intensité plus forte semble indiqué que les modèles en couches ne marchent pas [30]. Cependant cette augmentation d'intensité n'est pas nécessairement relié à une structure plus ordonnée [36]. Il a été suggéré que, avec l'augmentation de température, une relaxation des contraintes des arrangements entraînerait une augmentation des corrélations enter couches [26]. Il a été aussi considéré que dans les verres tels $\mathrm{SiO}_{2}$, les structure en couche étaient improbable. Gaskell et Wallis [32] ont utilisé les meilleurs modèles simulés pour le verre $\mathrm{SiO}_{2}$ [37] pour montrer que ces modèles contenaient des structures planes (quasi-plans de Bragg) qui étaient associées au pic à bas $\mathrm{Q}$. La présence d'une diffraction de Bragg à la même position que les structures à bas $\mathrm{Q}$ dans la plupart des verres a conduit Gaskell a proposé une explication générale : la réminiscence de quasi-plans de Bragg (distordus, imparfaits et pas nécessairement 
plans) dans les verres similaires à ceux présents dans les cristaux de composition proches. Ce modèle n'implique pas une structure des verres sous forme de couches et a des connections avec le modèle d'Elliott d'interférences entre le réseau et le vide. D'autre part, ce modèle a reçu le support de modélisations [38] et est relié avec des modèles de liquides [39]. Bien que ne traduisant pas un modèle de microcristallites, cette interprétation montrent les similitudes importantes entre verres et cristaux dans l'organisation à moyenne distance, ce qui a été également montré par l'analyse de fonctions de première différence dans les verres (voir sections suivantes).

\subsection{Le réseau polymérique}

La diffraction des neutrons est une des méthodes les plus employées pour étudier la structure des verres. En particulier, des informations structurales quantitatives sur les formateurs de réseau et le réseau polymérique peuvent être obtenues grâce aux fonctions de corrélation totale, dans lesquelles les paires associées à la matrice vitreuse sont dominantes. Les verres de silice et de bore ont été largement étudiés par cette méthode [40].

Dans les verres de phosphates, il a été possible de séparer les contributions entre les oxygènes pontants et non-pontants autour de $\mathbf{P}$ qui sont séparés de $0.1 \AA$, ce qui nécessite d'obtenir des données de diffraction jusqu'à $38 \AA^{-1}$ et donc d'avoir recours aux sources par spallation. De telles données fournissent des contraintes sur la polymérisation des chaînes de tétraèdres $\mathrm{PO}_{4}$ en fonction de la teneur en oxydes modificateur [41]. Le couplage diffraction des neutrons-diffraction des rayons X-Monte Carlo Inverse a permis de construire des modèles structuraux. La méthode de Monte Carlo Inverse (Reverse Monte Carlo, RMC) permet de générer des modèles atomistiques par ajustement des données expérimentales (voir section 5.2) [42]. Ces modèles ont montré par exemple que deux alcalins présent dans les verres étaient distribués de façon homogène [43]. L'ajout de sels dans ces verres (par exemple AgI [44]) conduit à la dilatation du réseau ce qui va favoriser la formation de chemins pour les déplacements ioniques.

Dans les verres boratés, l'accès à de grandes valeurs en $\mathrm{Q}$ a permis de discriminer deux distances correspondant au bore en coordinence triangulaire $\left(\mathrm{BO}_{3}\right)$ et au bore en coordinence tétraédrique $\left(\mathrm{BO}_{4}\right)$ [45]. Cette étude a permis de suivre le rapport $\mathrm{BO}_{3} / \mathrm{BO}_{4}$ en fonction de la composition et montre un excellent accord avec les expérience de RMC. Différentes études ont été menés sur les verres ioniques boratés ou phosphatés en combinant souvent diffraction des neutrons, des rayons $\mathrm{X}$ et modélisation par Monte Carlo Inverse. Il a été montré que l'ordre à moyenne distance du réseau $\mathrm{B}-\mathrm{O}$ diminuait avec une augmentation de la concentration en sel dopant et que, en présence de AgI, le réseau B-O forme une structure en chaîne avec les ions des sels reliant ces chaînes alors que, en présence des sels $(\mathrm{Li}, \mathrm{Na}))(\mathrm{Cl}, \mathrm{Br})$, les ions entrent dans le volume libre structurale et dilate le réseau boraté [46].

La méthode de substitution isotopique a aussi été utilisé pour extraire les différentes distributions de paires partielles dues au réseau vitreux. En particulier, dans un verre $\mathrm{GeSe}_{2}$ [47] toutes les paires ont pu être déterminées ce qui a permis de mettre en évidence des liaisons homopolaires. Des verres chalcogénures conducteurs ioniques ont été aussi largement étudié par cette méthode pour déterminer l'interaction entre le réseau et les sels ajoutés [48]. La structure des verres métalliques a été largement étudié également par diffraction des neutrons avec substitution isotopique [49]. 


\subsection{Les sites cationiques dans les verres}

L'ordre local autour des cations dans les verres nécessitent souvent le recours à des techniques spécifiques différentes de celles utilisées pour les structures cristallines. La diffraction anomale des rayons $\mathrm{X}$ et la diffraction des neutrons avec substitution isotopique sont des méthodes chimiquement sélectives, comme l'EXAFS, mais elles ont l'avantage de pouvoir sonder la structure sur de plus grandes distances (typiquement $10 \AA$ ) et avec une meilleure précision [50]. L'utilisation de matériaux substitués isotopiquement permet d'obtenir une information similaire à la diffraction anomale des rayons $\mathrm{X}$ mais pour des éléments différents, avec une meilleure statistique expérimentale et la possibilité d'extraire directement les distances cations-cations.

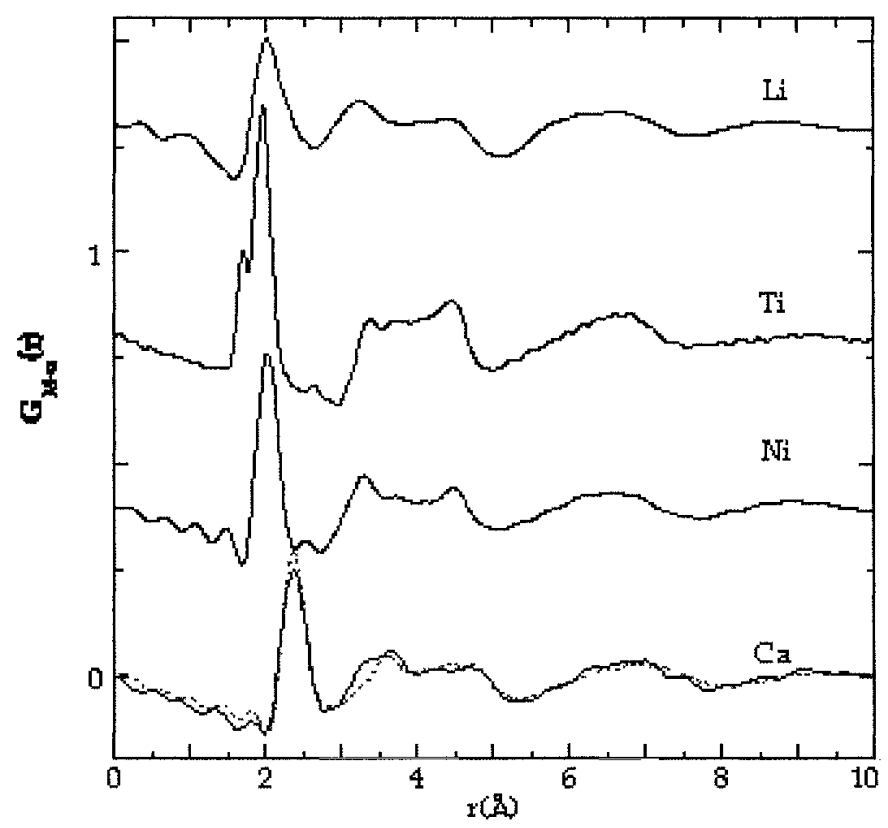

Figure 5 : Comparaison des fonctions de première différence, $\mathrm{GM}_{\mathrm{M}-\alpha}(\mathrm{r})$, pour $\mathrm{Li}$ dans $\mathrm{Li}_{2} \mathrm{O} \mathrm{Al}_{2} \mathrm{O}_{3} 2 \mathrm{SiO}_{2}$, Ti dans $\mathrm{K}_{2} \mathrm{OTiO}_{2} 2 \mathrm{SiO}_{2}$, Ni et Ca dans $2 \mathrm{CaONiO} 3 \mathrm{SiO}_{2}$ (de haut en bas) [D'après Ref. [52]].

Quelques exemples de fonctions de première différence obtenues par diffraction des neutrons pour des cations dans des verres silicatés et aluminosilicatés sont présentés sur la figure 5 [51]. L'intérêt de cette fonction peut être compris sur la figure 6 qui présente les fonctions de corrélations déterminées dans une étude complète sur un verre $\mathrm{K}_{2} \mathrm{OTiO}_{2} 2 \mathrm{SiO}_{2}$, avec substitution du Ti [52]. Dans la fonction de première différence, $G_{\mathrm{Ti}-\alpha}(r)$, nous observons que les corrélations Si-O et O-O présentant un poids important sont éliminées. Il apparaît alors que la première couche de voisins oxygènes autour de Ti peut être deconvoluée en deux distances distinctes Ti-O à 1.68 et $1.96 \AA$, qui permet de définir le site du Ti comme une pyramide à base carrée. Au contraire de l'EXAFS qui présente une seule distribution [53], la méthode de diffraction des neutrons avec substitution isotopique est capable de résoudre les deux distances 
Ti-O, grâce au large domaine en Q disponible qui donne une meilleure résolution dans l'espace réel.

Des similitudes importantes existent dans les fonctions présentées sur la figure 5, indépendamment de la concentration en cation (par exemple, $5.9 \%$ atomique pour $\mathrm{Ni}$ dans $\mathrm{Ca}_{2} \mathrm{NiSi}_{3} \mathrm{O}_{9}[54]$ vs. $14.3 \%$ atomique pour $\mathrm{Li}$ dans $\mathrm{LiAlSiO}_{4}$ [55]) ou de la matrice vitreuse (par exemple, $\mathrm{Li}$ dans un silicate, $\mathrm{Li}_{2} \mathrm{Si}_{2} \mathrm{O}_{5}[56]$ ou un aluminosilicate, $\mathrm{LiAlSiO}_{4}$ ). Des oscillations structurales sont discernables jusque vers $10 \AA$ dans les fonctions $G_{\mathrm{M}-\mathrm{a}}(\mathrm{r})$, ce qui indique un arrangement cationique très bien défini à moyenne distance.

Toutes les fonctions $\mathrm{G}_{\mathrm{M}-\mathrm{a}}(\mathrm{r})$ présentent un premier pic étroit qui résulte de la première couche de coordinence, indiquant des sites cationiques très bien définie dans les verres. Ces sites présentent des distances cation-oxygène et des coordinences qui peuvent être proches de celles observées dans des cristaux de compositions voisines $(\mathrm{Li}, \mathrm{Ti})$ pour les cations avec de faibles coordinences et un faible désordre radial. Cependant les cations avec des coordinences plus élevées présentent des nombres de coordinence plus faibles dans les verres que dans les cristaux. C'est le cas de $\mathrm{Ca}$ (coordinence 8 dans le cristal $\mathrm{CaNiSi}_{2} \mathrm{O}_{6}$ et coordinence 6 dans le verre $\mathrm{Ca}_{2} \mathrm{NiSi}_{3} \mathrm{O}_{9}$ ) et de $\mathrm{Ni}$ (coordinence 6 dans le cristal $\mathrm{CaNiSi}_{2} \mathrm{O}_{6}$ et coordinence 5 dans le verre $\mathrm{Ca}_{2} \mathrm{NiSi}_{3} \mathrm{O}_{9}$ ).

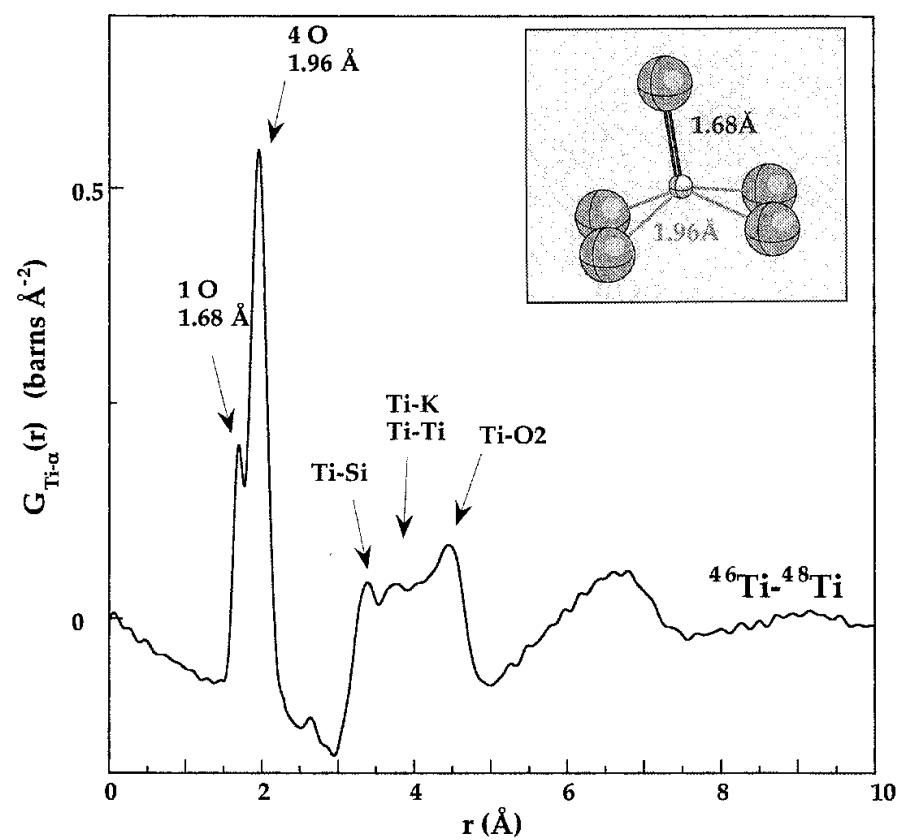

Figure 6 : Fonction de première différence pour $\mathrm{Ti}, \mathrm{G}_{\mathrm{Ti}}-\alpha(\mathrm{r})$. L'insert montre le site du titane.

Les effets de désordre radial peuvent être aussi quantifiés par diffraction des neutrons avec substitution isotopique. La principale corrélation cation-oxygène montre des déviations standards qui sont similaires pour tous les verres, $\sim 0.1 \AA$. Les données étendues en $Q$ permettent 
de très bien résoudre les géométries des sites. Le bon contraste des isotopes de Ti a permis de déconvoluer deux distances Ti-O séparées de $0.3 \AA$ dans le verre $\mathrm{K}_{2} \mathrm{OTiO}_{2}-2 \mathrm{SiO}_{2}$. Les distorsions de sites ont été aussi évaluées dans le cas de Li [54-57]. Le lithium est toujours tétracoordonné dans les verres d'oxydes mais des différences sont observées. L'environnement local autour de $\mathrm{Li}$ dans les verres aluminosilicatés est fortement distordu ( $3 \mathrm{O}$ à $2.02 \AA$ et $1 \mathrm{O}$ à $2.32 \AA$ ) avec une distorsion plus forte et une distance moyenne plus longue que pour les silicates. Des modélisations RMC des données de diffraction ont montrées que les tétraèdres $\mathrm{LiO}_{4}$ partagent des arêtes avec les tétraèdres $(\mathrm{Al}, \mathrm{Si}) \mathrm{O}_{4}$ dans le verre $\mathrm{LiAlSiO}_{4}$, ce qui implique des distances ( $\mathrm{Si}, \mathrm{Al})$ - $\mathrm{Li}$ courtes $(\sim 2.6-2.7 \AA)$ et des distances $\mathrm{Li}-\mathrm{O}$ longues. Ces distances et la distorsion des sites de $\mathrm{Li}$ dans $\mathrm{LiAlSiO}_{4}$ sont reliées au rôle de compensateur de charge de $\mathrm{Li}$ dans les aluminosilicates, contrairement à un rôle de modificateur de réseau dans les silicates où les tétraèdres $\mathrm{LiO}_{4}$ et $\mathrm{SiO}_{4}$ sont liés par sommets. Ces données reproduisent celles observées dans les cristaux de compositions voisines et montrent que la géométrie locale du site $\mathrm{Li}$ reflète des différences importantes de l'organisation structurale à plus longues distance. Une distribution asymétrique existe aussi pour $\mathrm{Ca}$ et $\mathrm{Ni}$ : la fonction $\mathrm{G}_{\mathrm{M}-\mathrm{a}}(\mathrm{r})$ présente une large contribution à grandes valeurs de $r$, bien qu'une exacte détermination en soit difficile.

\subsection{Ordre cationique à moyenne distance}

Entre 3 et $5 \AA$, toutes les fonctions $\mathrm{G}_{\mathrm{M}-\mathrm{a}}$ (r) présentent plusieurs contribution reliées aux seconds voisins (Figure 5). Ces pics sont proches des distances observées dans les cristaux de compositions voisines, ce qui montre que les verres conservent certaines caractéristiques structurales des cristaux. Cependant la présence de contributions au delà de $5 \AA$ dans les cristaux est contraire à un modèle de microcristallites [7]. Contrairement aux cristaux, on observe un déficit structural autour de 5-6 ̊̊ pour tous les cations étudiés. Les fonctions différentielles obtenues par diffraction anomale des rayons $\mathrm{X}$ présentent la même absence de corrélation, comme pour le $\mathrm{Sr}$ dans des verres silicatés [58].

Au delà de $5 \AA$, de large contributions centrées à 7 et $9 \AA$ sont observées et ne sont pas comparables aux distances attendues dans les cristaux. La distance de $5 \AA$ peut donc être considérée comme la taille limite des organisations structurales qui sont similaires dans les cristaux et les verres. Cependant, un ordre important persiste au delà de $5 \AA$ comme cela peut être révélé en calculant une seconde différence par diffraction des neutrons ou par des calculs de dynamique moléculaire [59].

\subsection{Distribution non-homogène des cations}

Les fonctions de double différence ont été obtenues pour le Ti [52], Ni [54], Ca [60] et $\mathrm{Li}$ [56] dans des verres silicatés [61]. Ces fonctions traduisent la distribution du cation dans le réseau vitreux et montrent des similitudes importantes. La figure 7 présente les fonctions de corrélation M-M.

La présence d'une première courte distance cations-cations indiquent une distribution non-homogène de ces éléments dans la structure du verre car une distribution homogène en considérant un arrangement compact de sphères donnerait une première distance cation-cation de $\sim 6 \AA$. La présence de domaine s'étendant jusque vers $9-10 \AA$ indique une organisation à moyenne distance. Pour tous les cations étudiés, si $R_{1}$ est la première distance $M-M$, la seconde apparaît proche de $\sqrt{3} R_{1}$ et la troisième proche de $\sqrt{7} R_{1}$. De plus, la distance à $\sqrt{2} R_{1}$ qui est 
caractéristique d'un arrangement tri-dimensionel des polyèdres est absente. Ces informations traduisent une organisation structurale des cations présentant un caractère fortement bidimensionel dans ces verres [20] et ont permis de remettre en cause le modèle historique de Zachariasen [4].

Des distances M-M similaires ont été observées par diffraction des rayons X dans des verres silicatés ou boratés contenant des éléments lourds dont les partiels et en particulier la paire $M-M$ va dominer les facteurs de structure expérimentaux. Ces études révèlent des corrélations cation-cation proche de 4,7 et $10 \AA$ aussi bien dans les verres silicatés silicate que dans les verres boratés [62], sur une très large gamme de composition (3-65 mol\% d'oxydes non-formateur). Un récente étude par diffraction anomale des rayons $\mathrm{X}$ au seuil $\mathrm{K}$ de $\mathrm{Sr}$ dans des verres silicatés a aussi montrée des distances $\mathrm{Sr}-\mathrm{Sr}$ à 4 et $7 \AA$ [63]. Ces structure hétérogène sont aussi fortifiés par des calculs numériques par Dynamique Moléculaire [58] ou RMC $[64,65]$, qui sont capables de reproduire des régions enrichies en cation.

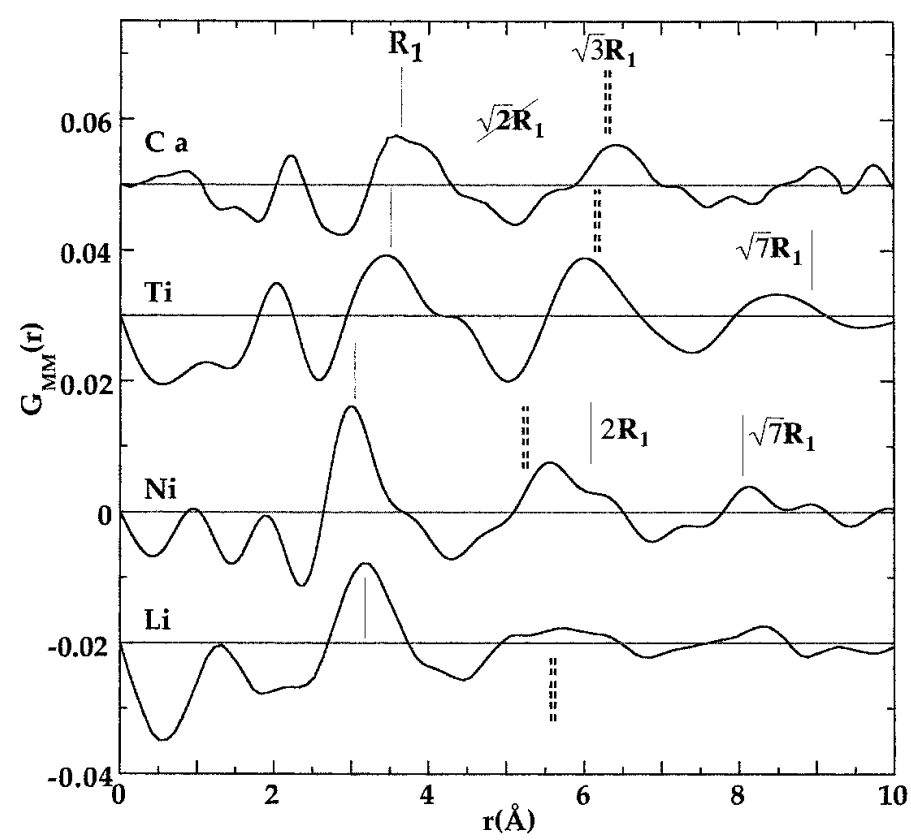

Figure 7: Distribution cation-cation, $\mathrm{G}_{\mathrm{MM}}(\mathrm{r})$, obtenues dans des verres : $\mathrm{Ca}$ dans $\mathrm{CaOSiO}_{2}$, Ti dans $\mathrm{K}_{2} \mathrm{OTiO} 22 \mathrm{SiO}_{2}$, Ni dans $2 \mathrm{CaONiO} 3 \mathrm{SiO}_{2}$ et $\mathrm{Li}$ dans $\mathrm{Li}_{2} \mathrm{O} 2 \mathrm{SiO}_{2}$ (de haut en bas), (D’après Réf. [61]). 


\section{APPORT DE LA DIFFRACTION DES RAYONS X ET DES SIMULATIONS}

En raison de l'anisotropie macroscopique des verres, une expérience de diffraction sur un verre donnera une fonction de corrélation à une dimension. Toute structure tri-dimensionelle à partir de ces données ne sera donc pas unique. Pour améliorer notre représentation structurale des verres, il est donc nécessaire de recourir à des techniques expérimentales variées et complémentaires (par exemple, diffraction des neutrons et des rayons $\mathrm{X}$, absorption des rayons $\mathrm{X}$ ). De plus, les différentes techniques de modélisations (Dynamique moléculaire classique ou $a b$ initio, Monte Carlo, Monte Carlo Inverse) permettent d'obtenir des modèles atomistiques tridimensionnels.

\subsection{Complémentarité de la diffraction des rayons $X$}

La diffraction des neutrons est souvent associée à la diffraction des rayons $\mathrm{X}$ pour obtenir des informations supplémentaires sur la structure des matériaux désordonnés. Ces deux méthodes permettent d'avoir accès à domaines étendus en moment de transfert et offre donc une bonne résolution des distances et du nombre de premier voisins. Ces deux techniques sont complémentaires car elles sont sensibles à différents éléments. Le couplage des deux méthodes de diffraction a été largement utilisé pour étudier des verres boratés ou phosphatés présentant une bonne conduction ionique. Le réseau vitreux boratés ou phosphatés a pu être étudié plus spécifiquement avec les résultats de diffraction des neutrons, alors que l'organisation des éléments responsables de la conduction ionique (oxydes alcalins ou sels) ont été regardé par diffraction des rayons $X[41,43-46,65]$.

\subsection{Complémentarité des simulations numériques}

En dépit du développement des techniques expérimentales, notre représentation de la structure des verres demeure partielle. Le recours à des simulations peut aider à mieux contraindre des modèles structuraux. Les techniques de dynamique moléculaire ou de Monte Carlo sont celles qui ont été le plus largement utilisées. La dynamique moléculaire permet de suivre, dans le temps et en fonction de la température, les mouvements d'un ensemble d'atomes interagissant au moyen d'un potentiel donné. On obtient ainsi des informations sur la dynamique du liquide, sur le comportement lors de la transition vitreuse ou sur la structure du verre. Cette méthode a été appliquée à un grand nombre de verres d'oxydes [66]. Cependant, pour des raisons de temps de calcul, les vitesses de trempe numériques sont considérablement plus importantes que celles obtenues expérimentalement. De plus, la détermination de potentiels représentant correctement les interactions atomiques est délicate. La méthode de Monte Carlo utilise des potentiels pour minimiser l'énergie du système mais elle a été assez peu utilisée [67]. Elle a par contre inspiré la technique de Monte Carlo Inverse (RMC) qui consiste à déplacer aléatoirement un ensemble d'atomes de façon à reproduire les données expérimentales, sans avoir recours à des potentiels inter atomiques [42].

L'interprétation des données de diffraction totale $(\mathrm{S}(\mathrm{Q}), \mathrm{G}(\mathrm{r}))$ est généralement difficile au delà des premiers voisins et, en particulier, pour les matériaux multicomposants. Au cours de la dernière décennie, la méthode de Monte Carlo Inverse (RMC) a été largement utilisé pour un ajustement quantitatif des données de diffraction obtenus sur des verres. Cette technique a permis d'obtenir des modèles atomistiques en accord avec les données de diffraction et les informations 
structurales extraites de ces modèles a permis de mieux comprendre la structure des verres. Le modèle initial est un paramètre essentiel dans les modélisations par RMC et habituellement, des configurations aléatoires ont été choisis. Cependant ces structures conduisent à des modèles avec des énergies entropiques très importantes et peu réalistes. La dynamique moléculaire offre des modèles de départ plus réalistes si des potentiels interatomiques existent, en dépit des limites de la méthode (temps de trempe expérimentalement pas atteignable). Même avec les meilleures simulations de dynamique moléculaire actuelles, des différences dans la position et l'intensité des pics existent avec les données expérimentales. Nous présentons sur la figure 8 la comparaison entre des données de diffraction de neutrons et de rayons $\mathrm{X}$ et des simulations de dynamique moléculaire obtenues sur un verre $55 \mathrm{CaO} 10 \mathrm{SiO}_{2} 35 \mathrm{Al}_{2} \mathrm{O}_{3}$, avec des potentiels de type Born-Mayer-Huggins [68]. Nous observons de petites différences, en particulier le pic vers 2.4 dans les fonctions de corrélation est imparfaitement reproduit.
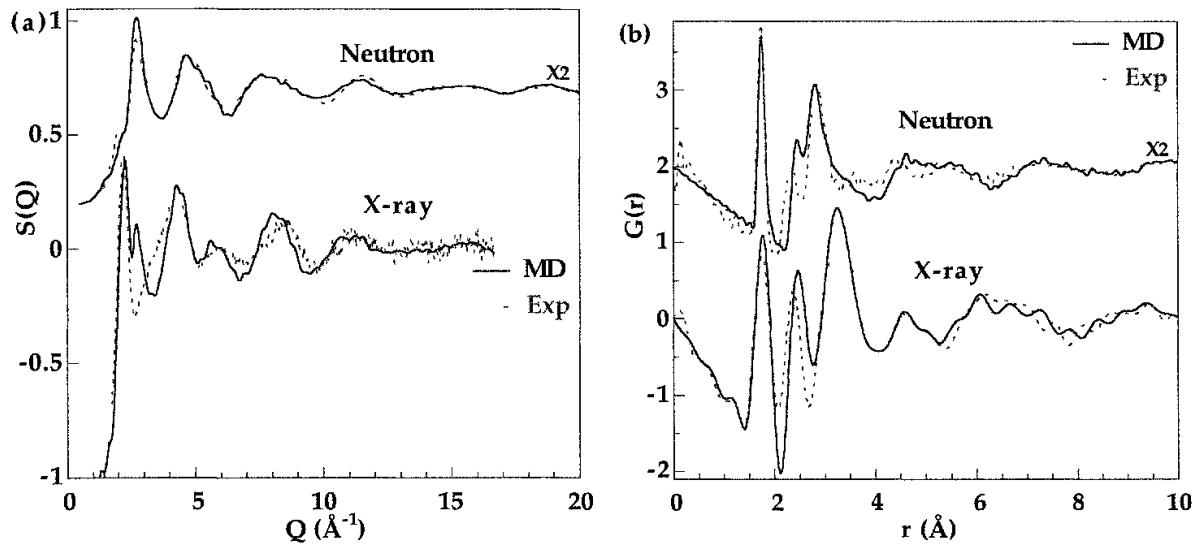

Figure 8 : (a) Facteurs de structure et (b) fonctions de corrélations obtenus expérimentalement par diffraction des neutrons et des rayons $\mathrm{X}$ (courbes hachés) et compares avec les résultats obtenues par dynamique moléculaire (courbes pleines) pour un verre $55 \mathrm{CaO} 10 \mathrm{SiO}_{2} 35 \mathrm{Al}_{2} \mathrm{O}_{3}$.

Ces modèles peuvent donc être ajustés en utilisant la méthode RMC pour obtenir des structures atomistiques en accord avec les grandeurs expérimentales. Le couplage entre expérience et simulations permet une meilleure interprétation des données expérimentales obtenues sur des verres multicomposants. Une approche nouvelle consiste également à utiliser les modèles ainsi ajustées par RMC pour améliorer les potentiels interatomiques. En effet, en calculant les distributions de paires partielles avant et après avoir appliquée la procédure RMC, on détermine les paires qui ont le plus varié et qui nécessitent donc une optimisation des paramètres de leur potentiel. La figure 9 compare la paire $\mathrm{Ca}-\mathrm{O}$ avant et après l'ajustement par RMC des données de la figure 8, ce qui met en évidence la nécessité d'avoir une plus faible distance $\mathrm{Ca}-\mathrm{O}$ pour un bon accord avec les données expérimentales. Ce type d'approche a conduit à la modification du potentiel de la paire $\mathrm{Ca}-\mathrm{O}$ [69]. 


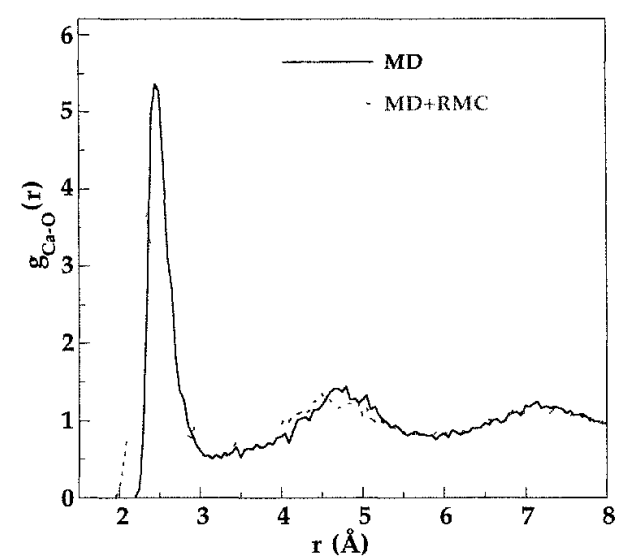

Figure 9 : Fonction de distribution de la paire Ca-O calculée par dynamique moléculaire (courbe pleine) et ajusté par RMC (courbe hachée). On remarque le déplacement du premier pic de $2.44 \AA$ par dynamique moléculaire à $2.35 \AA$ par ajustement RMC des données de diffraction.

L'ensemble des données expérimentales obtenues sur un verre peuvent être rationalisée en appliquant la méthode de modélisation RMC. Les différentes facteurs de structure partiels ou distribution de paires partielles peuvent ainsi être extraites pour un verre multicomposant. La figure 10 montre les résultats obtenus sur une verre $\mathrm{SrO}-2 \mathrm{SiO}_{2}$ par diffraction des neutrons (Figure 10.a) et diffraction anomale des rayons $\mathrm{X}$ au seuil $\mathrm{K}$ de $\mathrm{Sr}$ (Figure 10.b), qui a permis d'obtenir une fonction de première différence centrée sur le $\mathrm{Sr}$ (Figure 10.c). Cette exemple montre l'intérêt d'un couplage des deux méthodes de diffraction en raison de facteurs pondérant différents : la diffraction des neutrons est plus sensible aux paires associées avec le réseau silicatés et inversement la diffraction des rayons $\mathrm{X}$ va être fortement pondérée par les paires associées au Sr. Cette étude a permis de mieux comprendre les arrangements des cations Sr dans les verres et les similitudes présentées avec les cristaux de composition voisines [70]. L'analyse des fonctions de corrélations a permis de montrer que le strontium avait un environnement et une distribution différents dans la structure selon qu'il agissait en modificateur ou en compensateur de charge [65].

\section{CONCLUSIONS}

La diffraction des neutrons est une technique de choix pour étudier la structure des verres, notamment quand elle est associée avec la méthode de substitution isotopique. En plus de définir la couche de coordinence autour des cations dans les verres d'oxydes, elle donne une description précise du désordre radial des liaisons cation-oxygène grâce au large domaine en $\mathrm{Q}$ accessible. Les fonctions de premières différences obtenues par diffraction des neutrons autour des cations dans des verres silicatés montrent d'importantes similitudes dans l'organisation à moyenne distance. L'information la plus originale concerne la distribution des cations dans le verre qui peut être obtenue directement par la méthode de seconde différence. Le couplage de la diffraction des neutrons avec la diffraction des rayons $X$ et des simulations numériques est indispensable pour mieux contraindre les modèles structuraux des verres. 
(a)

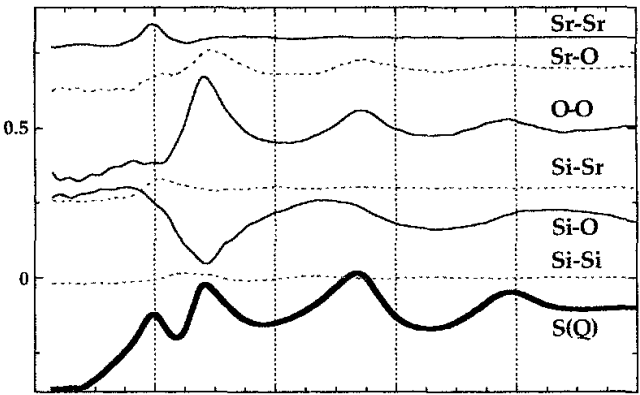

(b)

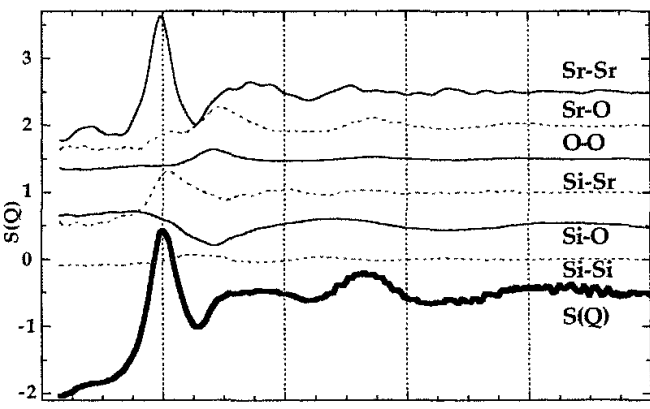

(c)

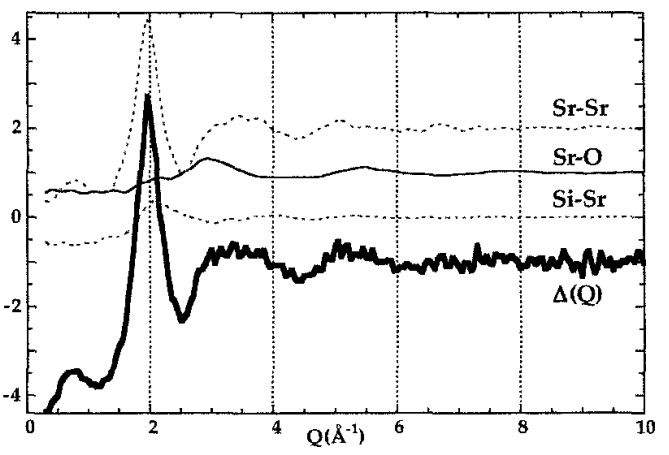

Figure 10 : Facteurs de structure totaux obtenus par (a) diffraction des neutrons, (b) diffraction des rayons $\mathrm{X}$ et (c) facteur de structure de première différence déterminé par diffraction anomale des rayons $\mathrm{X}$ au seuil $\mathrm{K}$ de $\mathrm{Sr}$ pour un verre $\mathrm{SrO}-\mathrm{SiO}_{2}$, comparés avec les facteurs de structure partiels pondérés obtenus par modélisation RMC. 


\section{Remerciements}

J'exprime toute ma reconnaissance à Georges Calas et Philip Gaskell pour leur contribution importante dans une partie des travaux présentés dans ce manuscrit. Alan Soper, Alex Hannon, Brigitte Bouchet-Fabre, Sophie Creux, Robert Bellissent sont remerciés pour l'aide apporté dans l'acquisition et le traitement des données à ISIS, au LLB et au LURE. Je remercie Robert Mc Greevy pour l'utilisation du programme RMCA et Jean-Marc Delaye et Dominique Ghaleb pour les calculs de dynamique moléculaire. Henry Fischer est remercié pour la relecture attentive de ce manuscrit.

\section{Références}

1. P. H. Gaskell, J. Non-Cryst. Solids 222, 1 (1997) ; P. H. Gaskell, J. Non-Cryst. Solids 293295, $146(2001)$.

2. A. C. Wright, J. Non-Cryst. Solids 106, 1 (1988).

3. S. R. Elliott, Nature, 354, 445 (1991).

4. W. H. Zachariasen, J. Amer. Chem. Soc. 54, 3841 (1932).

5. G. N. Greaves, A. Fontaine, P. Lagarde, D. Raoux, S. J .Gurman, Nature, 293, 611 (1981) ; G. Calas,J. Petiau, Bull. Min. 106, 33 (1983).

6. G. E. Brown, Jr., F. Farges, G. Calas, in Structure, dynamics, and properties of silicate melts, edited by J. F. Stebbins, P. F. McMillan, D. B. Dingwell, (Mineralogical Society of America, Washington, 1995), Vol. 32, p. 317.

7. M. C. Eckersley, P. H. Gaskell, A. C. Barnes, P. Chieux, Nature 335, 525 (1988).

8. P. H. Gaskell, in Materials Science and Technology, edited by R. W. Cahn, P. Haasen, E. J. Craner (VCH, Weinheim, 1991), Vol. 9, p. 175 ; P. H. Gaskell, J. Zhao; G. Calas, L. Galoisy, in The physics of non-crystalline solids, edited by L. D. Pye, W. C. LaCourse, H. J. Stevens (Taylor \& Francis, London, 1992), p. 53 ;P. H. Gaskell, in Methods in determination of partials structure factors of disordered matter by neutron and anomalous $X$-ray diffraction, edited by J. B. Suck, P. Chieux, D. Raoux, C. Rielke (World Scientific Publ. Co., Singapore, 1993), p. 34.

9. G. N. Greaves, Phil. Mag. B 60, 793 (1989).

10. C. H .L. Goodman, Phys. Chem. Glasses 26, 1 (1985).

11. M. D. Ingram, Phil. Mag. B 60, 729 (1989) ; M. D. Ingram, J. Non-Cryst. Solids 131-133, 955 (1991).

12. J. C. Phillips, C. A. Beevers, S. E. B. Gould, Phys. Rev. B 21, 5274 (1980).

13. P.H. Gaskell, Nature 289474 (1981) ; J. M. Dubois, P.H. Gaskell, G. Le Caer, Proc Roy. Soc. (Lond.) A402, 323 (1985).

14. A. C. Wright, in Advances in structure research by diffraction methods (Pergamon Press, 1974), Vol. 5, p. 1 ; A. C. Wright, A. J. Leadbetter, Phys. Chem. Glasses 17 (1976) ; A. C. Wright, J. Non-Crsyt. Solids 106, 1 (1988).

15. P. Chieux, in Neutron diffraction, edited by H. Dachs (Springer-Verlag, Berlin, 1978).

16. T. E. Faber, J. M. Ziman, Phil. Mag. 11, 153 (1965).

17. E. Lorch, J. Phys. C: Solid St. Phys. 2229 (1969).

18. H. F. Poulsen, J, Neuefeind, H.-B. Neumann, J. R. Schneider, M. D. Zeilder, J. Non-Cryst. Solids 188, 63 (1995).

19. J. E. Enderby, D. M. North, P. A. Egelstaff, Phil. Mag., 14 (1966) 961.

20. P. H. Gaskell, M. C. Eckersley, A. C. Barnes, P. Chieux, Nature 350, 675 (1991).

21. H. Schlenz, Kirfel, A., Schulmeister, K., Wartner, N., Mader, W., Raberg, W., Wandelt, K., Oligschleger, C., Bender, S., Franke, R., Hormes, J., Hoffbauer, W., Lansmann, V., Jansen, M., zotov, N., Marian, C., Putz, H., Neuefeind, J., J. Non-Cryst. Solids 297, 37 (2001).

22. R. N. Sinclair, Anomalous neutron diffraction studies of disordered materials, In Methods in determination of partial structure factors of disordered matter by neutron and anomalous X-ray diffraction, Ed. J.B. Suck, P. Chieux, D. Raoux, C. Rielke, World Scientific, Singapore (1993) 107. 
23. J. E. Enderby, P. M. N. Gullidge, In Methods of experimental physics, Eds. D.L. Price, K. Sköld, Academic Press, London, 23 Part B (1987) 471.

24. H. Tsutsu, K. Tamura, H. Endo, Solid State Commun. 52, 877 (1984) ; K. Tanaka, J. NonCryst. Solids 90, 363 (1987).

25. K. Tanaka, Phil. Mag. Lett. 57, 183 (1988).

26. L. E. Busse, S. R. Nagel, Phys. Rev. Lett. 47, 1848 (1981) ; L. E. Busse, Phys. Rev B 29, 3639 (1984).

27. M. Misawa, J. Chem. Phys. 91, 5648 (1989).

28. M. Misawa, D. L. Price, K. Suzuki, K., J. Non-Cryst. Solids 37, 85 (1980).

29. D. L. Price, S. C. Moss, R. Reijers, M.-L. Saboungi, S. Susman, S., J Phys. C: Solid State Phys. 21, L1069 (1988).

30. S. R. Elliott, Phys. Rev. Lett. 67, 711 (1991). S. R. Elliott, J. Phys.:Cond. Matter 4, 7661 (1992).

31. E. A. Chechetkina, Solid Stat. Comm. 91, 101 (1994).

32. P. H. Gaskell, D. J .Wallis, Phys. Rev. Lett. 76, 66 (1996).

33. M. Misawa, J. Chem. Phys. 93, 6774 (1990).

34. S. Veprek, H. U. Beyeler, Phil. Mag. 44, 557 (1981) ; T. G. Fowler, S. R. Elliott, J. NonCryst. Solids 92, 31 (1987) ; J. Dixmier, J. Phys. 1 France 2, 1011 (1992).

35. J. Blétry, Phil. Mag. B 62, 469 (1990).

36. J. C. Phillips, A. C. Beevers, S. E. B. Gould, Phys. Rev. B 21, 5274 (1980) ;J. C. Phillips, J. Non-Cryst. Solids 43, 37 (1981) ; L. Cervinka, O. Smotlacha, L. Tichy, J. Non-cryst. Solids 97 \& 98, 183 (1987) ; L. Cervinka, J. Non-Cryst. Solids 90, 371 (1987).

37. L. F. Gladden, J. Non-Cryst. Solids 119, 318 (1990) ; J. D. Wicks, Studies of disordered materials, Ph.D. thesis, Oxford, 1993.

38. A. Le Bail, J. Non-Cryst. Solids 183, 39 (1995).

39. M. Wilson, P. A. Madden, Phys. Rev. Lett. 72, 3033 (1994) ; R. Fayos, F. J. Bermejo, J. Dawidowski, H. E. Fischer, M. A. González, Phys. Rev. Lett. 77, 3823 (1996).

40. A. C. Hannon, D. I. Grimley, R. A. Hulme, A. C. Wright, R. N. Sinclair, J. Non-Cryst. Solids 177, 299 (1994) ; M. Misawa, J. Non-Cryst. Solids 122, 33 (1990) ; A.C. Wright, J. Non-Cryst. Solids 179, 84 (1994).

41. U. Hoppe, G. Walter, R. Kranold, D. Stachel, J. Non-Cryst. Solids 263\&264, 29 (2000).

42. R. L. McGreevy, Nucl. Inst. Meth. Phys. Res. A 354, 1 (1995) ; R. L. McGreevy, J. Phys: Condens. Matter 13, R877 (2001) ; R. L. Mc Greevy, P. Zetterström, J. Non-Cryst. Solids 293-295, 297 (2001).

43. J. Swenson, A. Matic, C. Karlsson, L. Börjesson, Phys. Rev. B 63 (2001).

44. J. Swenson, A. Matic, C. Gejke, L. Börjesson, W. S. Howells, M. J. Capitan, Phys. Rev. B 60, 12023 (1999).

45. J. Swenson, L. Börjesson, W. S. Howells, Phys. Rev. B 52, 9310 (1995).

46. J. Swenson, L. Börjesson, W. S. Howells, Phys. Rev. B 57, 13514 (1998).

47. I. Petri, P. S. Salmon, H. E. Fischer, Phys. Rev. Lett. 84, 2413 (2000).

48. C. J. Benmore, P. S. Salmon, Phys. Rev. Letters 73, 264 (1994) ; J. Liu, P. S. Salmon, Europhys. Letters 39, 521 (1997) ; P. S. Salmon, S. Xin, Phys. Rev. B 65, 64202 (2002) ; J. H. Lee, A. Pradel, G. Tiallades, M. Ribes, S. R. Elliott, Phys. Rev. B 56, 10934 (1997).

49. P. Lamparter, Phys. Scripta T57, 45 (1995).

50. L. Cormier, S. Creux, L. Galoisy, G. Calas, P. H. Gaskell, Chem. Geol. 128, 77 (1996).

51. L. Cormier, P. H. Gaskell, G. Calas, A. K. Soper, Phys. Rev. B 58, 11322 (1998).

52. F. Farges, G. E. Brown, Jr., A. Navrotsky, H. Gan, J. J. Rehr, Geochim. Cosmochim. Acta 60, 3055 (1996).

53. P. H. Gaskell, J. Zhao,G. Calas, L. Galoisy, in The physics of non-crystalline solids, edited by L. D. Pye, W. C. LaCourse, H. J. Stevens (Taylor \& Francis, London, 1992), p. 53.

54. L. Cormier, P. H. Gaskell, G. Calas, J. Zhao, A. K. Soper, Phys. Rev. B 57, 8067 (1998).

55. J. Zhao, P. H. Gaskell, M. M. Cluckie, A. K. Soper, J. Non-Cryst. Solids 232-234, 721 (1998).

56. H. Uhlig, M. J. Hoffmann, H. P. Lamparter, F. Aldinger, R. Bellissent, S. Steeb, J. Amer. Ceram. Soc. 79, 2833 (1996). 
57. S. Creux, B. Bouchet-Fabre, P. H. Gaskell, J. Non-Cryst. Solids 192 \& 193, 360 (1995).

58. M. C. Abramo, C. Caccamo, G. Pizzimenti, J. Chem. Phys. 96, 9083 (1992).

59. P. H. Gaskell, M. C. Eckersley, A. C. Barnes, P. Chieux, Nature 350, 675 (1991).

60. L. Cormier, L. Galoisy, J.-M. Delaye, D. Ghaleb, G. Calas, C.R. Acad. Sci., série IV 2, 249 (2001).

61. L. Cormier, L. Galoisy, J.-M. Delaye, D. Ghaleb, G. Calas, C.R. Acad. Sci., série IV 2, 249 (2001).

62. C. Brosset, Phys. Chem. Glasses 4, 99 (1963) ; C. D. Hanson, T. Egami, J. Non-Cryst. Solids 87, 171 (1986) ; J. Krogh-Moe, Phys. Chem. Glasses 3, 208 (1962) ; S. Block, G. J. Piermarini, Phys. Chem. Glasses 5, 138 (1964) ; I. Yasui, H. Hasegawa, Y. Suito, J. NonCryst. Solids 106, 30 (1988) ; I. Yasui, H. Hasegawa, Y. Saito, Y., Akasaka, J. Non-Cryst. Solids 123, 71 (1990).

63. S. Creux, B. Bouchet-Fabre, P. H. Gaskell, J. Non-Cryst. Solids 192 \& 193, 360 (1995).

64. L. Cormier, G. Calas, P. H. Gaskell, J. Phys. Cond. Mat. 9, 10129 (1997).

65. L. Cormier, G. Calas, S. Creux, P. H. Gaskell, B. Bouchet-Fabre, A. C. Hannon, Phys. Rev. B 59, 13517 (1999).

66. P. H. Poole, P. F. McMillan, G. H. Wolf, in Structure, dynamics and properties of silicate melts, edited by J. F. Stebbins, P. F. McMillan, D. B. Dingwell (Mineralogical Society of America, Washington, 1995), Vol. 32 ;B. Vessal, M. Amini, C. Richard, C. R. A. Catlow, M. Leslie, Trans. Am. Cryst. Assoc 27, 15 (1991) ; M. C. Abramo, G. Pizzimenti, A. Consolo, Philos. Mag. B 64, 495 (1991) ; W. Soppe, C. Van der Marel, H. W. den Hartog, J. Non-Cryst. Solids 101, 101 (1988).

67. V. K. Schiff, J. Non-Cryst. Solids 123, 36 (1990).

68. L. Cormier, D. Ghaleb, D. Neuville, J.-M. Delaye, G. Calas, J. Phys. Chem. (soumis).

69. J.-M. Delaye, L. Cormier, D. Ghaleb, G. Calas, J. Non-Cryst. Solids 293-295, 290 (2001).

70. L. Cormier, P. H. Gaskell, S. Creux, J. Non-Cryst. Solids 248, 84 (1999). 